RPI-97-N122; WM-97-116; hep-ph/9801205

\title{
Perturbative Duality in the Resonance Spin Structure Functions
}

\author{
Carl E. Carlson \\ Physics Department, College of William and Mary, Williamsburg, VA 23187 \\ Nimai C. Mukhopadhyay \\ Department of Physics, Applied Physics, and Astronomy, Rensselaer Polytechnical Institute, Troy, NY 12180-3590
}

(31 Decembar 1997)

\begin{abstract}
We investigate the relations between the spin structure functions in the scaling and resonance regions. We examine the possible duality between the two, and draw inferences for the behavior of the asymmetry $A_{1}$ at large $x$. Finally, we point out the importance of additional polarized structure function data in the resonance region in terms of testing the hysteresis of perturbative physics.
\end{abstract}

Duality is a relation between the deep inelastic scattering region and the resonance region in lepton hadron scattering [1]. It is a manifestation of the fact that the single quark reaction rate determines the scale of the reaction rate for the entire process down to remarkably low energies and momentum transfers. In the resonance region, final state interactions are crucial and all quarks must be involved in the reaction. Nevertheless, the overall reaction rate is still determined by the single quark reaction rate - provided we average over regions comparable to the widths of the resonances. So far, these inclusive/exclusive connections have been seen to work for the measured [2,3] unpolarized structure functions [1, 1 . Precious little experimental information is available for the rôle of duality for the polarized structure functions, although efforts in this direction are beginning to bear fruit [5].

Here we investigate the relevance and consequences of duality for the polarized structure functions. We shall show that perturbative QCD arguments lead to an inclusive/exclusive relationship in the polarized case akin to that in the unpolarized one. Also, we shall discuss perturbative expectations for resonance contributions in the high $x$ limit of the polarization asymmetry. Finally, we note interesting features of the present data and point out what can be learned from additional polarized structure function data in the resonance region at higher $Q^{2}$.

We begin with some definitions and kinematic relations. For deep inelastic scattering, $e+p \rightarrow e+X$, the structure functions $W_{i}$ and $G_{i}$ are defined by

$$
\begin{aligned}
W_{\mu \nu} & =\frac{1}{4 \pi m_{N}} \sum_{X}(2 \pi)^{4} \delta^{4}\left(q+p-p_{X}\right) \\
& \times\left\langle p s\left|j_{\mu}(0)\right| X\right\rangle\left\langle X\left|j_{\nu}(0)\right| p s\right\rangle \\
& =-g_{\mu \nu} W_{1}+\frac{1}{m_{N}^{2}} p_{\mu} p_{\nu} W_{2}+\frac{i}{m_{N}} \epsilon_{\mu \nu \lambda \sigma} q^{\lambda} \times \\
& \times\left[s^{\sigma} G_{1}+\frac{1}{m_{N}^{2}}\left(p \cdot q s^{\sigma}-s \cdot q p^{\sigma}\right) G_{2}\right],
\end{aligned}
$$

where $s$ is the spin vector of the nucleon target and satisfies $s \cdot p=0$ and $s^{2}=-1, p$ is the momentum of the target nucleon, and $q$ is the momentum of the incoming virtual photon. Often, the structure functions are replaced by

$$
\begin{aligned}
\nu W_{2} & =F_{2} \quad, \quad m_{N} W_{1}=F_{1} \quad, \\
\nu G_{1} & =g_{1} \quad, \quad \frac{\nu^{2}}{m_{N}} G_{2}=g_{2}
\end{aligned}
$$

where it is expected that $F_{i}$ and $g_{i}$ depend just on $x \equiv$ $Q^{2} / 2 m_{N} \nu$ (where $Q^{2}=-q^{2}$ ) in the scaling region, up to logarithmic corrections.

The exclusive process $e+p \rightarrow e+R$, where $R$ stands for the final baryon, a resonance or the nucleon (in the case of elastic scattering), is described by the helicity amplitudes,

$$
G_{m}=\frac{1}{2 m_{N}}\left\langle R, \lambda^{\prime}=m-\frac{1}{2}\left|\epsilon_{\mu}^{(m)} \cdot j^{\mu}(0)\right| N, \lambda=\frac{1}{2}\right\rangle .
$$

The photon polarization vectors are

$$
\begin{aligned}
\epsilon^{( \pm)} & =(0, \mp 1,-i, 0) / \sqrt{2}, \\
\epsilon^{(0)} & =\frac{1}{Q}(|\vec{q}|, 0,0, \nu),
\end{aligned}
$$

with $q=(\nu, 0,0,|\vec{q}|)$.

Note that the helicity of the final baryon is

$$
\begin{aligned}
& \frac{1}{2} \text { for } G_{+}, \\
- & \frac{1}{2} \text { for } G_{0}, \\
- & \frac{3}{2} \text { for } G_{-} .
\end{aligned}
$$

Thus if the final baryon has spin $1 / 2, G_{-}$must be absent. For elastic scattering, the helicity amplitudes are related to well-known form factors by

$$
\begin{aligned}
G_{+} & =\frac{Q}{m_{N} \sqrt{2}} G_{M}, \\
G_{0} & =G_{E} .
\end{aligned}
$$

For the non-elastic case, one often uses the amplitudes

$$
\left|A_{1 / 2,3 / 2}\right|=e \sqrt{\frac{m_{N}}{m_{R}^{2}-m_{N}^{2}}}\left|G_{+,-}\right|
$$


where $e$ is the proton charge $(0.3028 \ldots)$.

For a single sharp resonance $R$ the relations between the structure functions and the helicity amplitudes are:

$$
\begin{aligned}
& F_{1}= m_{N}^{2} \delta\left(W^{2}-m_{R}^{2}\right)\left[\left|G_{+}\right|^{2}+\left|G_{-}\right|^{2}\right], \\
&\left(1+\frac{\nu^{2}}{Q^{2}}\right) F_{2}= m_{N} \nu \delta\left(W^{2}-m_{R}^{2}\right) \\
& \times\left[\left|G_{+}\right|^{2}+2\left|G_{0}\right|^{2}+\left|G_{-}\right|^{2}\right], \\
&\left(1+\frac{Q^{2}}{\nu^{2}}\right) g_{1}= m_{N}^{2} \delta\left(W^{2}-m_{R}^{2}\right) \\
& \times\left[\left|G_{+}\right|^{2}-\left|G_{-}\right|^{2}+(-1)^{s_{R}-\frac{1}{2}} \eta_{R} \frac{Q \sqrt{2}}{\nu} G_{0}^{*} G_{+}\right], \\
&\left(1+\frac{Q^{2}}{\nu^{2}}\right) g_{2}=-m_{N}^{2} \delta\left(W^{2}-m_{R}^{2}\right) \\
& \times\left[\left|G_{+}\right|^{2}-\left|G_{-}\right|^{2}-(-1)^{s_{R}-\frac{1}{2}} \eta_{R} \frac{Q \sqrt{2}}{\nu} G_{0}^{*} G_{+}\right],
\end{aligned}
$$

where $W^{2} \equiv(p+q)^{2}$, the total hadronic mass squared, and $s_{R}$ and $\eta_{R}$ are the spin and parity of the resonance. The delta function for the sharp resonance can be most simply approximated by

$$
\begin{aligned}
\delta\left(W^{2}-m_{N}^{2}\right) & \approx \frac{1}{2 m_{R}} \frac{\Gamma_{R} / 2 \pi}{\left(W-m_{R}\right)^{2}+\Gamma_{R}^{2} / 4} \\
& \stackrel{\text { peak }}{\longrightarrow} \frac{1}{\pi m_{R} \Gamma_{R}},
\end{aligned}
$$

with $\Gamma_{R}$ being the width of the resonance.

Let us now discuss the scaling properties of $G_{ \pm, 0}$ and of $g_{1,2}$. The resonance contributions to the structure functions fall with increasing $Q^{2}$ and also move to progressively higher $x$, approaching $x=1$. They thus may be described as falling with $x$, at a certain rate. We wish to determine if the fall-off rate is the same as that in the deep inelastic region as $x \rightarrow 1$, but at much higher $Q^{2}$. This is already known to be true for the spin independent structure functions, and the phenomenon is known as Bloom-Gilman duality [1]. Hence, we will concentrate our attention on the spin dependent structure functions.

The counting rules [6] give the following behavior at high $Q^{2}$ for the helicity amplitudes (modulo logarithms) 迎:

$$
\begin{aligned}
& G_{+}=g_{+} / Q^{3}, \quad G_{0}=\left(m_{N}\right) g_{0} / Q^{4}, \\
& G_{-}=\left(m_{N}\right)^{2} g_{-} / Q^{5},
\end{aligned}
$$

where $g_{ \pm, 0}$ are constants, real in leading Born order, and the mass factors are put in purely for dimensional reasons. This allows us to find the resonance contribution to $g_{1}$ at the resonance peak and at high $Q^{2}$ to be,

$$
g_{1}=\frac{m_{N}^{2}}{\pi m_{R} \Gamma_{R}} \frac{g_{+}^{2}}{Q^{6}}=\frac{m_{N}^{2}}{\pi m_{R} \Gamma_{R}} \frac{g_{+}^{2}}{\left(m_{R}^{2}-m_{N}^{2}\right)^{3}}(1-x)^{3} .
$$

The second result requires

$$
\frac{1}{Q^{2}}=\frac{1}{W^{2}-m_{N}^{2}} \frac{1-x}{x} \approx \frac{1}{m_{R}^{2}-m_{N}^{2}}(1-x)
$$

for $x \rightarrow 1$ and $W \approx m_{R}$. Similarly,

$$
g_{2}=-\frac{m_{N}^{2}}{\pi m_{R} \Gamma_{R}} \frac{(1-x)^{3}}{\left(m_{R}^{2}-m_{N}^{2}\right)^{3}} g_{+}\left(g_{+}-\frac{\eta_{R}(-1)^{s_{R}-\frac{1}{2}}}{\sqrt{2}} g_{0}\right),
$$

for high $Q^{2}$. It is interesting to note that the high $Q^{2}$ resonance contributions to the polarized structure functions can be inferred from unpolarized structure function measurements since $g_{+}$and $g_{0}$ can be separately obtained from transverse and longitudinal scattering. If $g_{2}$ is small, as seems to be indicated [7], then there must be a relation between the transverse and longitudinal resonance form factors, viz.,

$$
\sqrt{2} g_{+} \approx \eta_{R}(-1)^{s_{R}-\frac{1}{2}} g_{0} .
$$

In the deep inelastic region, the spin structure function $g_{1}$ is related to the quark distributions as a manner similar to $F_{1}$ except for one sign,

$$
\begin{aligned}
& g_{1}=\frac{1}{2} \sum e_{q}^{2}\left(q_{\uparrow}\left(x, Q^{2}\right)-q_{\downarrow}\left(x, Q^{2}\right)\right), \\
& F_{1}=\frac{1}{2} \sum e_{q}^{2}\left(q_{\uparrow}\left(x, Q^{2}\right)+q_{\downarrow}\left(x, Q^{2}\right)\right) .
\end{aligned}
$$

The $q_{\uparrow, \downarrow}$ are the quark distributions for quark helicities parallel or antiparallel to the parent nucleon polarization. Perturbative QCD dictates that $q_{\uparrow}$ dominates as $x \rightarrow$ 1 [8]. If so, the $x \rightarrow 1$ behavior will be the same for both functions. Even if pQCD did not work for the $x \rightarrow 1$ limit, it would require a remarkable cancellation to make the high $x$ behavior different for $F_{1}$ and $g_{1}$. Since the behavior of $F_{1}$ is well-known, we can conclude

$$
\lim _{x \rightarrow 1} g_{1}(x) \propto(1-x)^{3}
$$

in the deep inelastic region. This is the same as the contribution from the resonance region. One part of the duality between the resonance and deep inelastic regions is thus established.

It is less clear what the deep inelastic result should be for $g_{2}$. There is no unique parton model formula for it. However the twist-two form of the Wandzura-Wilczek relation [9],

$$
g_{2}(x)=-g_{1}(x)+\int_{x}^{1} d x^{\prime} \frac{g_{1}\left(x^{\prime}\right)}{x^{\prime}},
$$

leads to the result that

$$
\lim _{x \rightarrow 1} g_{2}(x)=-g_{1}(x) \propto(1-x)^{3} .
$$

Thus the scaling part of the duality is established for $g_{2}$ also. 
Now we shall further examine the $x \rightarrow 1$ behavior of $g_{1}$, or of the photon asymmetry $A_{1}$,

$$
A_{1} \equiv \frac{\sigma_{1 / 2}-\sigma_{3 / 2}}{\sigma_{1 / 2}+\sigma_{3 / 2}}=\frac{g_{1}-\frac{Q^{2}}{\nu^{2}} g_{2}}{F_{1}},
$$

where the cross sections are for photon absorption with initial state spin projections of $1 / 2$ and $3 / 2$. For a resonance,

$$
A_{1}=\frac{\left|G_{+}\right|^{2}-\left|G_{-}\right|^{2}}{\left|G_{+}\right|^{2}+\left|G_{-}\right|^{2}} .
$$

For the elastic point, $x=1$, there is only the nucleon and $G_{-}=0$ so that rigorously

$$
A_{1}(x=1)=1 \text {. }
$$

For a single resonance of spin $1 / 2$, the same is true. Even for spin $3 / 2$ and higher resonances, the scaling rules tell us that $\left|G_{+}\right|>>\left|G_{-}\right|$at high $Q^{2}$, so that $A_{1} \rightarrow 1$ as $x \rightarrow 1$. If the only backgrounds under a given resonance are due to tails of other resonances, then the same rule still applies,

$$
\lim _{Q^{2} \rightarrow \infty} A_{1}=1 \quad \text { if only resonant background. }
$$

However, if the non-resonant background is dominated by Born terms [10], we can get $A_{1} \rightarrow 1$ anyway. In the resonance region the $t$-channel and $u$-channel diagrams have propagators that suppress their contributions at high $Q^{2}$, leaving the $s$-channel diagram which has only $\sigma_{1 / 2}$. (Purely as an aside, a dominant $t$-channel Born term is needed for certain measurements of the pion form factor, and this can indeed happen even at high $Q^{2}$, but only if the final hadronic state is well outside the resonance region.) Note that the isospin of the resonance plays no rôle in the above considerations.

The $x \rightarrow 1$ behavior of $A_{1}$ in the scaling region can be got from the ratio of the two eqns. (14). We quote the results for $\mathrm{pQCD}$, where the $x \rightarrow 1$ results for the polarized quark distributions are mentioned above); for $\mathrm{SU}(6)$, where no distinction is made between the distributions of differently polarized quarks; and for a number of modern suggestions for the polarized quark distributions [11]. One has

$$
\lim _{x \rightarrow 1} A_{1}=\left\{\begin{array}{cl}
1 & \text { pQCD, or Soffer et al. [11] } \\
\frac{5}{9} & \text { SU(6), } \\
0.75 & \text { GS, version B 11] } \\
0.66 & \text { GRSV ("standard" NLO) 11] }
\end{array}\right.
$$

The last two are given at their respective benchmark $Q^{2}$.

Let us look at the present relevant data on the polarized structure functions in the resonance region. There is a paucity of such data. The SLAC measurements, from the E143 collaboration recently [5] (and from earlier data with larger error bars 12 ), do cover $W^{2}<5 \mathrm{GeV}^{2}$ at $Q^{2} \approx 0.5$ and $1.2 \mathrm{GeV}^{2}$. These $Q^{2}$ are still too low for a duality test, since duality is not working at these $Q^{2}$ in the spin-independent case [1, 1 . Nevertheless it is useful to discuss the data that exists.

In the first resonance region, the $\Delta(1232)$ itself gives nearly all the signal at very low $Q^{2}$ in the unpolarized case and may be expected to do the same in the polarized case. Further, at low $Q^{2}, \Delta$ electroproduction is dominated by the magnetic dipole amplitude [13,14], which leads to $\left|G_{-}\right| \approx \sqrt{3}\left|G_{+}\right|$and

$$
A_{1}\left(\Delta, \text { low } Q^{2}\right) \approx-1 / 2 \text {. }
$$

Abe et al. find for $Q^{2} \approx 0.5 \mathrm{GeV}^{2}$ and in the $\Delta$ region, that $A_{1} \approx-1 / 3$, in qualitative agreement with our expectation. However, for $Q^{2} \approx 1.2 \mathrm{GeV}^{2}$, the measured value of $A_{1}$ is consistent with zero (albeit also consistent with $-1 / 2$ at a $2 \sigma$ level). Since there is evidence that the M1 dominance is still valid for the resonance itself, the $A_{1}$ result must be due to the background and resonance giving approximately canceling contributions. This suggests a violation of the strict construction of Bloom-Gilman duality, since the $Q^{2}$ dependence of the resonance and background do not match. However, $Q^{2}$ is still low.

It is reminiscent of the unpolarized case, where for the $\Delta$ Bloom-Gilman duality works (above a few $\mathrm{GeV}^{2}$ ) in the sense of the resonance region average matching the scaling curve, and does so without having the resonance to continuum ratio be constant, but rather because of an interplay between resonance and continuum [4]. As one falls, the other rises, relative to the scaling curve, and the sum stays about the same. So as in the unpolarized case, the sum over channels allows matching the scaling curve at low $Q^{2}$, perhaps so in the polarized case the sum over channels will show the perturbative polarization prediction at a lower $Q^{2}$ when a single channel will not.

For the second resonance region, the prediction for $A_{1}$ involves the $S_{11}(1535)$ and $D_{13}(1520)$, as well as the nonresonant background. At very low $Q^{2}$, the largest resonant amplitude is the $A_{3 / 2}$ for exciting the $D_{13}$ [16], the next largest is the $A_{1 / 2}$ for the $S_{11}$ [17], and $A_{1 / 2}$ for the $D_{13}$ is quite small. However, the resonances soon reconcile themselves to the high $Q^{2}$ expectations, as for the $D_{13}$ the $A_{1 / 2}$ and $A_{3 / 2}$ change relative size in the vicinity of $1 \mathrm{GeV}^{2}$ [18]. Hence, considering the resonant contributions alone, we expect $A_{1}$ to be negative at low $Q^{2}$ and become positive before $1 \mathrm{GeV}^{2}$. The available data 5] show $A_{1}$ to be positive at both 0.5 and $1.2 \mathrm{GeV}^{2}$.

For polarized structure functions, in contrast to the unpolarized case, duality must break down spectacularly at low enough $Q^{2}$ [19]. The argument goes by considering the Ellis-Jaffe integral [20], written as

$$
\int_{\nu_{0}}^{\infty} \frac{d \nu}{\nu^{2}} g_{1}^{p}=\frac{2 m_{N} \Gamma^{p}}{Q^{2}}
$$

with $\Gamma^{p}$ approximately constant at high $Q^{2}$, and comparing it to the Drell-Hearn-Gerasimov sum rule [21], which 
may be written in the form

$$
\int_{\nu_{0}}^{\infty} \frac{d \nu}{\nu^{2}} g_{1}^{p}\left(\nu, Q^{2}=0\right)=-\frac{\kappa_{p}^{2}}{2 m_{N}} .
$$

Above, $\kappa_{p}$ is the anomalous magnetic moment of the proton, and $\nu_{0}$ is given by the pion production threshold. The quantity $\Gamma_{p}$ is measured to be positive at $Q^{2}$ of several $\mathrm{GeV}^{2}$ (see, for example, [5]). If $g_{1}$ were on the average the same at very low $Q^{2}$ as it is at high $Q^{2}$, then the right hand side of the Drell-Hearn-Gerasimov sum rule would be positive - and it is clearly not.

We have shown that polarization structure function data in the resonance region at higher $Q^{2}$ are interesting and can throw significant new light on the issue of duality. Facilities like Jefferson Lab, SLAC, and HERMES can all contribute over a significant range of $Q^{2}$ and $W$. The idea that the single quark cross section sets the scale on the average even in the resonance region gets a new field of exploration in the polarized structure function. Unlike the unpolarized case, one expects its breakdown at sufficiently low $Q^{2}$. Finding this breakdown will signal the onset of a region where the final state interactions obliterate even a remnant of perturbative physics.

We thank R. M. Davidson, Keith Griffioen, and P. Stoler for useful comments, and members of the Jefferson Lab Hall A collaboration for stimulating discussions. CEC thanks the NSF for support under grant PHY-9600415; and NCM is grateful to the U. S. Department of Energy for its support through grant DE-FG0288ER40448.

[1] E. D. Bloom and F. J. Gilman, Phys. Rev. Lett. 25 (1970) 1140; Phys. Rev. D 4 (1971) 2901.

[2] P. Stoler, Phys. Rev. Lett. 66 (1991) 1003; Phys. Rev. D 44 (1991) 73; Phys. Rep. 226, 103 (1993); G. Sterman and P. Stoler, Ann. Rev. Nucl. and Part. Sci. (in press).

[3] P. E. Bosted et al., Phys. Rev. D 49, 3091 (1994); C. Koeppel, Ph.D. thesis (American University, 1994).

[4] C.E. Carlson and N.C. Mukhopadhyay, Phys. Rev. D 41 (1990) R2343; Phys. Rev. D 47 (1993) R1737; Phys. Rev. Lett. 74, 1288 (1995).

[5] K. Abe et al., Phys. Rev. Lett. 78, 815 (1997).

[6] S. J. Brodsky and G. Farrar, Phys. Rev. Lett. 31, 1153 (1973), Phys. Rev. D 11, 1309 (1975); V. A. Matveev, R. M. Muradyan, and A. N. Tavkhelidze, Lett. Nuovo Cimento 7, 719 (1973).

[7] K. Abe et al., Phys. Lett. B 404, 377 (1997); K. Abe et al., Phys. Rev. Lett. 76, 587 (1996); D. Adams et al, Phys. Lett. B 336, 125 (1994).

[8] G. R. Farrar and D. R. Jackson, Phys. Rev. Lett. 35, 1416 (1975).

[9] S. Wandzura and F. Wilczek, Phys. Lett. 72B, 195 (1977); R. Jaffe, Comments Nucl. Part. Phys. 19, 239 (1990).
[10] R. M. Davidson, N. C. Mukhopadhyay, and R. S. Wittman, Phys. Rev. D 43, 71 (1991).

[11] F. Buccella and J. Soffer, Phys. Rev. D 48, 5416 (1993); C. Bourrely and J. Soffer, Phys. Rev. D 53, 4067 (1996); T. Gehrmann and W. J. Stirling, Phys. Rev. D 53, 6100 (1996); M. Glück, E. Reya, M. Stratmann, and W. Vogelsang, Phys. Rev. D 53, 4775 (1996).

[12] G. Baum et al., Phys. Rev. Lett. 45, 2000 (1980).

[13] V. D. Burkert and L. Elouadrhiri, Phys. Rev. Lett. 75, 3614 (1995).

[14] R. M. Davidson and N. C. Mukhopadhyay, Phys. Lett. 353B, 151 (1995).

[15] C. E. Carlson and J. L. Poor, Phys. Rev. D 38, 2758 (1988); G. R. Farrar, H. Zhang, A. Oglublin, and I. R. Zhitnitsky, Nucl. Phys. B. 311, 585 (1989); J. Bonekamp, Bonn report (1989).

[16] Particle Data Group, R. M Barnett et al., Phys. Rev. D 54, 1 (1996).

[17] B. Krusche et al., Phys. Lett. 397B, 171 (1997); N. C. Mukhopadhyay, J.-F. Zhang, and M. Benmerrouche, Phys. Rev. Lett. 75, 3022 (1995).

[18] V. Burkert in Research Program at CEBAF, 1986, ed. Franz Gross (CEBAF, Newport News, 1986).

[19] F. Close, in Excited Baryons 1988, Proc. of the Topical Workshop on Excited Baryons, G. Adams, N. C. Mukhopadhyay, and P. Stoler, editors [World Scientific, Singapore, 1989]; V. D. Burkert and B. L. Ioffe, J. Exp. Theor. Phys. 78, 619 (1994).

[20] J. Ellis and R. Jaffe, Phys. Rev. D XX, xxx, 1973; see also J. D. Bjorken, Phys. Rev. 148, 1467 (1966); D 1, 1376 (1970).

[21] S. D. Drell and A. C. Hearn, Phys. Rev. Lett. 16, 908 (1966); S. B. Gerasimov, Sov. J. Nucl. Phys. 2, 598 (1966). 